\title{
Stereo-Vision Mobile Robotics Navigation through Metric-Topological Learning
}

\section{Ebrahim Mattar*}

MIET, MIEEE, College of Engineering, University of Bahrain, Bahrain

\section{Summary}

Autonomous mobile robots should be capable to learn and maintain visual models of their environments. Maps are needed for path-planning, self-localization, and human-robot interaction. In contrary, stereo-vision based mobile robot navigation has always been a challenge. This is due to complexity of integrating massive amount of visual information gathered by visual system, while undergoing a motion. Visual perception of an environment, is also an important capability for mobile robots, where lots of efforts are being undertaken by mobile robots research community. Once it comes to integrating environment visual information for clever navigation maps learning, the issue gets even much complicated. Therefore, we shall highlight few recent attempts to close such gap between visual sensory, and AI related navigation maps learning.

To start with, literature has indicated that, AI related mobile robot navigation has been under research since early 1990s, this is when Pan et al. have put their research findings back on 1995, that was related to the use of "FUZZY NAV: A Vision Based Robot Navigation Architecture using Fuzzy Inference for Uncertainty Reasoning”,[1]. They reported a robust vision based control architecture for indoor mobile robot navigation. The architecture takes advantage of the high throughput of neural networks for the processing of camera images. It employs fuzzy logic to deal with the uncertainty in the inferences drawn from the vision data. They stated that, "the sixteen fuzzy terms that have proved sufficient for the desired navigational behavior", and "the architecture we present in this paper allows our robot to simultaneously navigate and avoid obstacles both static and dynamic".

Another important topic to mention is the well-received manuscript related to development in visual navigation as early 2000s. The literature has indicated that Guilherme and Avinash [2], have collectively put a manuscript that surveys the late developments over the last 20 years within the field of vision-based mobile robot navigation, obviously before 2002. They stated that, "For indoor robots in structured environments, we have dealt separately with the cases of geometrical and topological models of space. For unstructured environments, we have discussed the cases of navigation using optical flows, using methods from the appearance-based paradigm, and by recognition of specific objects in the environment". In 2003, Petru et al. [3], also laid research results related to Behavior-Based Neuro-Fuzzy Controller for Mobile Robot Navigation. They indicated that, "Behavior learning methods are used to solve complex control problems that autonomous robots encounter in an unfamiliar real-world environment. Neural networks, fuzzy logic, and reinforcement and evolutionary-learning methods can be used to implement basic behavioral functions". Control system was organized in a top-bottom hierarchy. Once multiple lowlevel behaviors are required, command fusion was used to combine the output of several Neuro-fuzzy sub-systems. Hence, a switching coordination technique selects a suitable behavior from the set of possible higher level behaviors. In addition, enhancing mobile robots stereo vision navigation is becoming a necessity once maps learning is also needed to learn the navigation environments. Specifically for much complicated tasks that depend entirely on visual data. Research directions have been also directed towards maps learning. Ho-Dong et al. [4] have introduced an augmented reality mobile robot based vision system. They introduce a tele-presence vision system for monitoring of a network based mobile robot. The vision system is a vision part of human machine interface with augmented reality for the network based mobile robot. They synchronize head motion of human user and the camera motion of the mobile robot using visual information, user of the mobile robot can monitor environment of the mobile robot as eyesight of mobile robot.

Filliata \& Meyer [5], stated, "map-based navigation systems are categorized according to three-level hierarchy of localization strategies, respectively call upon direct position inference, single-hypothesis tracking, and multiple hypothesis tracking". A typical review of ANN approach was also reported by Filliata \& Meyer [5]. In [6], Araujo has presented Fuzzy ART Neural architecture for mobile robot map learning and navigation. Araujo proposed methods that integrated into a navigation architecture.

Araujo [6], also stated that, "with the new navigation architecture the mobile robot is able to navigate in changing worlds, and a degree of optimality is maintained, associated to a shortest path planning approach implemented in real-time over the underlying global world model". For demonstrating the feasibility and effectiveness of the proposed methods, a number of experimental results were also attained for a (Nomad 200) mobile robotic system. Development and integration of generic components for a teachable vision-based mobile robot is introduced by Tomohiro et al. [7]. In their efforts, they present a mobile robotic system for human assistance in navigationthe robot navigates by receiving visual instructions from a human being and is able to replicate them autonomously. They describe three generic components defined as HOST, VISION, and CONTROL components as well as their integration in our teachable mobile robot. Each component is described with a peculiar feature of extensibility. Especially in the VISION component, there are two major features. The first one is a correlator which each vision board possesses. The correlator does block-matching between the template and the grabbed images in real-time. The other is the PIM library which manages the visual tasks over limited parallel visual resources of the mobile robot. Skill acquisition of a ball lifting task using a mobile robot with a monocular vision system was also introduced by Ryosuke et al. [8]. The work presents a basic examination of skill acquisition of a ball lifting

*Corresponding author: Ebrahim Mattar , MIET, MIEEE, College of Engineering University of Bahrain, Bahrain, Tel: 973 1787 6606; E-mail: ebmattar@IEEE.org

Received July 21, 2014; Accepted July 22, 2014; Published July 25, 2014

Citation: Mattar E (2014) Stereo-Vision Mobile Robotics Navigation through MetricTopological Learning. Adv Robot Autom 3: e123. doi: 10.4172/2168-9695.1000e123

Copyright: (c) 2014 Mattar E. This is an open-access article distributed under the terms of the Creative Commons Attribution License, which permits unrestricted use, distribution, and reproduction in any medium, provided the original author and source are credited. 
task using a mobile robot with a monocular vision system. Ball lifting is considered as a basic practice in sports such as tennis or soccer. They examine the performance of a robot system and demonstrate that the robot performs the task in spite of many difficulties. The purpose is to develop an intelligent robot system that performs a human skill, they have built a basic but significant robot system as a first step. The various table text styles are provided. The formatter will need to create these components, incorporating the applicable criteria that follow.

Mobile robot self-localization in complex indoor environments using monocular vision and 3D model was moreover presented by Andreja et al. [9]. In their research, they consider the problem of mobile robot pose estimation using visual information from a single camera and odometry readings. The focus was on building composite environmental maps and models, fast online rendering, real-time complex and noisy image segmentation. The 3D model of the robot's environment was constructed using a professional freeware computer graphics tool and pre-stored in the memory of the robot's on-board computer. Estimation of mobile robot pose as a stochastic variable was completed by correspondences of image lines, extracted using Random Window Randomized Hough Transform line detection algorithm, and model lines, predicted using odometry readings and 3D environment model. Platon et al. [10], used (GRBF) Generalized Radial Basis Function. Hence, the produced mapping was linked in a mathematical way with standard robotic probabilistic map-building tools and Neurofuzzy systems. This enabled direct interpretation of the map data and its exploitation for the control of mobile robot movement.

In $\mathrm{Al}$ Mutib et al. [11] have proposed a description of a research outcomes for building visual maps for KSU-IMR navigation intelligence. That was based on implementing a multi-level control for intelligence. It was implemented with specific navigation behaviors that are desirable for enhanced maps interaction and navigating. Specifically they elaborated on details of an Upper-Level of mobile intelligence which was based on mixing a number of functionaries. KSU-IMR mobile robot three levels of intelligence were based on using (Fuzzy System for wheels control), (ANN for Mid-Level SLAM), and (PCA mixed with Neuro-Fuzzy decision based architecture) for Upper-Level of mobile maps learning and intelligence (Figure 1).

\section{References}

1. Pan J, Pack DJ, Kosaka A, Kak AC (1995) FUZZY-NAV: A Vision Based Robot Navigation Architecture using Fuzzy Inference for Uncertainty Reasoning. Proceedings of the World Congress on Neural Networks 2: 602-607.

2. Guilherme D and Avinash K (2002) Vision for Mobile Robot Navigation: A Survey. IEEE Transactions on Pattern Analysis and Machine Intelligence 24 237-266.

3. Petru R, Emil MP, Thom EW, Aurel C, Hans JWS (2003) Behavior-Based Neuro-Fuzzy Controller for Mobile Robot Navigation. IEEE Transactions on Instrumentation and Measurement 52: 1617-1622.

4. Ho-Dong, Dongwon K, Min-Chul P, Gwi Tae P (2008) Augmented reality based vision system for network based mobile robot. Proceedings of ComputerHuman Interaction - $8^{\text {th }}$ Asia-Pacific Conference 5: 123-130.

5. Filliata D and Meyer J (2003) Map-based navigation in mobile robots: I. A review of localization strategies. Cognitive Systems Research 4: 243-282.

6. Araujo R (2006) Prune-Able Fuzzy ART Neural Architecture for Robot Map Learning and Navigation in Dynamic Environments. IEEE Transactions on Neural Network 17: 1235-1249.

7. Tomohiro S, Yoshio M, Taichi K, Masayuki I, Hirochika I (1996) Developmen and integration of generic components for a teachable vision-based mobile robot. IEEE/ASME Transactions on Mechatronics 1: 230-236.

8. Ryosuke M, Fumiaki T, Fumio M (2006) Skill acquisition of a ball lifting task using a mobile robot with a monocular vision system. IEEE International Conference on Intelligent Robots and Systems.

9. Andreja K, Sanjin B, Ivan P (2007) Mobile robot self-localization in complex indoor environments using monocular vision and 3D model. IEEE/ASME International Conference on Advanced Intelligent Mechatronics.

10. Platon AP and Elpida ST (2002) Robotic Navigation Through Neurofuzzy Maps Mimicking the Human Hippocampus. Proceedings of $10^{\text {th }}$ Mediterranean Conference on Control and Automation, Lisbon.

11. Al Mutib K, Mattar E, AISulaiman M, Ramdane H (2014) PCA Based Mobile Robot Navigation and Mapping Intelligence. 27 $7^{\text {th }}$ International Conference on Computer Applications in Industry and Engineering, New Orleans, Louisiana, USA.

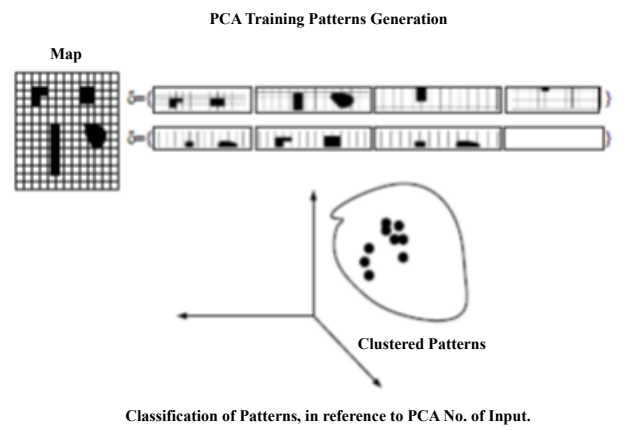

Figure 1: "KSU-IMR" PCA reduction to und7 erstand maps, [11] 